\title{
Assistante médicale, quo vadis?
}

\author{
Le futur de l'assistante médicale est incertain. Quelles seront ses tâches en I'an \\ 2020 si cette profession existe encore?
}

\section{Michel Marchev}

Président des délégués cantonaux aux questions des assistantes médicales
Correspondance: Dr Michel Marchev

Président des délégués aux questions des assistantes médicales

Elfenstrasse 18

CH-3000 Berne 15

\section{Scénario no 1: \\ les conditions de travail continuent à se dégrader pour les médecins de premier recours...}

Il est trop tard pour relancer la médecine de premier recours. Le dernier cabinet du médecin de famille traditionnel se situe sur le Ballenberg et l'on considère la pratique de la médecine générale comme un hobby que seuls les fils et les filles de bonne familles ou les femmes médecins dont le mari a un bon salaire peuvent exercer.

En raison des baisses répétées du point tarifaire, la pratique du laboratoire au cabinet médical coûte quelque Fr. 20 000.- par an aux généralistes.

La licence pour les radiographies coûte Fr. 10 000.- par an et à cela s'ajoutent les frais de différents examens coûteux d'assurance qualité des appareils et leurs utilisateurs. Pour des raisons de radioprotection, les appareils radiologiques ne peuvent en principe être maniés uniquement par des techniciennes diplômées en radiologie médicale (TRM) selon un système efficace de maîtrise du taux de radiation. Etant donné que l'exposition aux radiations est nettement moins nuisible dans les instituts de radiologie, il va de soi qu'à l'avenir, c'est là que ces examens seront pratiqués.

En outre et pour plus de facilité, les factures seront directement établies par les assureursmaladie. Les réclamations pénibles et autres demandes de remboursement seront ainsi reléguées aux oubliettes.

Il va donc de soi que la profession d'assistante médicale a considérablement changé

La pratique du laboratoire et les radiographies font désormais partie du passé. La tâche principale des assistantes médicales consiste dorénavant, outre l'accueil des patients, à gérer les différents formulaires hautement diversifiés de transfert des patients ainsi que les ordonnances. Il incombe aux assistantes de choisir le formulaire approprié et de le mettre à disposition de son supérieur au moment voulu, accompagné du stylo spécial prévu à cet effet.
Le généraliste dispose de formulaires de transfert faciles à utiliser pour les radiographies, les électrocardiogrammes et le laboratoire. Pour l'envoi de patients à des spécialistes reconnus, les caisses-maladie proposent des formulaires en ligne spécialement conçus à cet effet où il suffit d'indiquer le numéro d'assurance-maladie et les organes à examiner. Le choix du spécialiste le moins onéreux se fait directement par les gestionnaires de cas («case-manager») des assureurs. Le choix des médicaments destinés aux patients relève des pharmaciens et des spécialistes des caisses-maladie. Sur le formulaire conçu à cet effet, il suffit d'indiquer le diagnostic selon le code tessinois.

On ne trouve pratiquement plus d'assistantes médicales bénéficiant d'une vaste et solide formation et le fait que les généralistes ont, pour des raisons financières, renoncé à la pratique du laboratoire et des radiographies à leur cabinet, a entraîné une baisse des places d'apprentissage, ce qui a complètement asséché le marché. En outre, les coûts salariaux ont énormément augmenté et atteignent désormais quelque Fr. 120 000.- par an.

\section{Scénario no 2: \\ I'opinion d'un éternel optimiste ...}

Les médecins de premier recours sont choyés, les politiques et les fonctionnaires des assurances ont enfin compris que les généralistes sont irremplaçables et qu'ils contribuent de façon décisive à réduire les coûts. Etant donné que les représentants de cette profession se font rares, des programmes sont mis sur pied pour rendre la profession plus attrayante. On se souvient aussi que les médecins de premier recours, grâce à leur laboratoire d'analyses, aux radiographies, à leurs connaissances en matière d'électrocardiogrammes et de spirométrie, étaient à même de régler 80 à $85 \%$ des problèmes médicaux de la population. Le fait qu'ils soient proches de la population et leur connaissance du contexte familial et professionnel du patient en ont fait des médecins à part. Cette proximité avec le patient pré- 
sente de nombreux avantages, notamment de fournir des soins avantageux à la population, et a été scientifiquement prouvée dans une étude de l'Université de St-Gall parue en 2015. Cette étude critique également violemment que ce soit justement ce pilier des soins médicaux de base qui fait les frais de la détérioration des conditions économiques au début du XXI ${ }^{\mathrm{e}}$ siècle.

L'étude relève également que ces généralistes sont secondés par des assistantes médicales disposant de solides connaissances techniques pour la pratique du laboratoire, les radiographies, les électrocardiogrammes, la spirométrie et les tâches administratives. En outre et grâce à leurs compétences en matière de communication, leur sensibilité face aux personnes malades et leur immense talent d'organisation elles permettent aux médecins de premier recours d'exercer leur activité avec succès.

En 2016, le Conseil fédéral décide, se fondant en cela sur les résultats de l'étude, d'ordonner des mesures urgentes en faveur de la médecine de premier recours et d'une formation plus étendue des assistantes médicales. Suite à cette décision, le Parlement met à disposition dans les trois mois les moyens financiers nécessaires à ce projet.

\section{D'après quel scénario nous faut-il former les futures assistantes médicales?}

Il convient d'en décider maintenant car les contenus de formation et d'examen doivent être définis lors de la révision du règlement d'apprentissage des assistantes médicales. Cette révision est obligatoire dans le cadre du remaniement de la plupart des professions OFFT régies par la nouvelle loi sur la formation professionnelle.

Tout souhait, suggestion ou autre idée par rapport à la révision de la réglementation est la bienvenue et doit être envoyée au service des assistantes médicales du Secrétariat général de la FMH.

P.-S.: J'espère que la pétition du CMPR en faveur de la médecine de premier recours et la manifestation des généralistes du $1^{\mathrm{er}}$ avril 2006 sur la Place fédérale permettront d'ores et déjà de poser les jalons en faveur de la réalisation du second scénario. Je vous invite à faire acte de présence le $1^{\mathrm{er}}$ avril prochain, car il est important de marquer notre position.

Vous trouverez des informations à l'adresse suivante: www.sgam.ch. 